\title{
Symmetry energy at subnuclear densities and nuclei in neutron star crusts
}

\author{
Kazuhiro Oyamatsu ${ }^{1,2}$ and Kei Iida ${ }^{2,3,4}$ \\ ${ }^{1}$ Department of Media Theories and Production, Aichi Shukutoku University, Nagakute, Nagakute-cho, Aichi-gun, Aichi 480-1197, Japan \\ ${ }^{2}$ The Institute of Physical and Chemical Research (RIKEN), Hirosawa, Wako, Saitama 351-0198, Japan \\ ${ }^{3}$ RIKEN BNL Research Center, Brookhaven National Laboratory, Upton, New York 11973-5000, USA \\ ${ }^{4}$ Department of Materials Science, Kochi University, Akebono-cho, Kochi 780-8520, Japan
}

(Received 3 October 2006; published 5 January 2007)

\begin{abstract}
We examine how the properties of inhomogeneous nuclear matter at subnuclear densities depend on the density dependence of the symmetry energy. Using a macroscopic nuclear model we calculate the size and shape of nuclei in neutron star matter at zero temperature in a way dependent on the density dependence of the symmetry energy. We find that for smaller symmetry energy at subnuclear densities, corresponding to the larger density symmetry coefficient $L$, the charge number of nuclei is smaller and the critical density at which matter with nuclei or bubbles becomes uniform is lower. The decrease in the charge number is associated with the dependence of the surface tension on the nuclear density and the density of a sea of neutrons, whereas the decrease in the critical density can be generally understood in terms of proton clustering instability in uniform matter.
\end{abstract}

DOI: 10.1103/PhysRevC.75.015801

PACS number(s): 97.60.Jd, 26.60.+c, 21.65.+f

\section{INTRODUCTION}

The inner crust of a neutron star consists of a Coulomb lattice of nuclei embedded in a roughly uniform neutralizing background of electrons and in a sea of neutrons [1]. The equation of state (EOS) of uniform nuclear matter and the surface tension, both at large neutron excess, are essential to understanding of the equilibrium properties of matter in the crust. However, laboratory data on nuclei reflect only the bulk and surface properties of nearly symmetric nuclear matter [2,3]. So far, calculations of the equilibrium properties of matter in the crust depend on the way to extrapolate the known bulk and surface properties to large neutron excess, which is different among earlier investigations [1].

In this article we systematically analyze the question of how the equilibrium properties of inhomogeneous nuclear matter at subnuclear densities depend on the parameter characterizing the density dependence of the symmetry energy. In doing so, we utilize a macroscopic nuclear model [2] in which the equilibrium nucleon distribution depends on the EOS of nuclear matter through minimization of the energy density functional. One of the most important quantities is the charge number of the equilibrium nuclide. In a liquid-drop picture [1], this charge number is determined by the size equilibrium condition that controls the ratio between the Coulomb and surface energies. This condition tells that the charge number squared is proportional to the surface tension and the nuclear volume. As we shall see, the density dependence of the symmetry energy, which controls the surface tension by affecting the nuclear density and the density of the neutron gas, controls the charge number as well.

We also address the question of how matter with nuclei or bubbles melts into uniform matter with increasing density. In this melting process, rodlike and slablike nuclei embedded in a gas of neutrons, as well as rodlike and roughly spherical neutron-gas regions (bubbles) surrounded by a nucleon liquid, are expected to occur [4-7]. At a density where roughly spherical nuclei are so closely packed that they occupy about
$1 / 8$ of the system volume, the nuclei tend to be elongated and eventually fuse into nuclear rods. The advantage of this rod formation is a reduction in the total surface area from the roughly spherical case. However, whether bubbles and nonspherical nuclei actually appear in neutron star crusts depends on the critical density at which proton clustering instability occurs in uniform nuclear matter [8]; they are expected to appear when the density corresponding to the nuclear volume fraction of about $1 / 8$ is smaller than the critical density for proton clustering. We find that this critical density is in turn controlled by the symmetry energy at subnuclear densities.

Earlier investigations on such exotic nuclei are more or less based on specific nuclear models [1,9]. An exception is the work by Watanabe et al. [10], which is systematic in the sense that the liquid-drop model calculations were performed in a way that was dependent on the proton chemical potential in pure neutron matter, $\mu_{p}^{(0)}$ and the surface tension. It was found that the density at which the system dissolves into uniform matter increases with increasing $\mu_{p}^{(0)}$. However, it remains to be clarified why some nuclear models $[6,11,12]$ predict the absence of bubbles and nonspherical nuclei. It is important to note that these models predict relatively high pressure for pure neutron matter (or, equivalently, relatively small symmetry energy) at densities around half the normal nuclear density, whereas the work by Watanabe et al. [10] used a parametrization [13] based on the microscopic calculations by Siemens and Pandharipande [14] as the EOS of pure neutron matter and fix the density dependence of the symmetry energy. This parametrization is consistent with the recent Green's function Monte Carlo (GFMC) calculations [15] at neutron densities up to about half the normal nuclear density. We will give a unified picture about the presence of bubbles and nonspherical nuclei by describing the pressure of pure neutron matter in terms of the density dependence of the symmetry energy.

The size and shape of nuclei in the crust bear relevance to the thermal and rotational evolution of neutron stars. This 
is because thermal conductivity and neutrino emissivity in the crust are controlled by electron-nucleus scattering [16], whereas the motion of superfluid neutron vortices in the crust is affected by vortex-nucleus interactions [17]. However, real crustal matter has to be accompanied by defects and impurities, which can play a more important role in the star's evolution [18]. In a real neutron star, furthermore, the nuclear system is more or less out of equilibrium in the course of mass accretion onto the surface of the star and the star's spin-down [19-21]. Such disordered and nonequilibrium properties are beyond the scope of this article.

In Sec. II, we construct a model for inhomogeneous nuclear matter at subnuclear densities in a way dependent on the EOS of nearly symmetric nuclear matter near the saturation point. The equilibrium size and shape of nuclei at given density are then calculated from the model constructed in Sec. III. Section IV is devoted to evaluations of the critical density at which uniform matter becomes unstable against proton clustering. Our conclusions are given in Sec. V.

\section{MODEL FOR MATTER AT SUBNUCLEAR DENSITIES}

In this section, we construct a macroscopic model for zerotemperature, $\beta$-equilibrated, inhomogeneous nuclear matter at subnuclear densities. This is an extension of Ref. [2] to the case in which a gas of dripped neutrons is present, which is based on Ref. [7]. Here we focus on how macroscopic properties of the system depend on the EOS of nearly symmetric nuclear matter and, for simplicity, ignore various effects such as nucleon pairing effects [22], shell effects in inhomogeneous matter [23,24], fluctuation-induced displacements of nuclei and bubbles [10], and electron screening effects [25].

The bulk energy per nucleon is an essential ingredient of the macroscopic nuclear model. We set this energy as

$$
\begin{aligned}
w= & \frac{3 \hbar^{2}\left(3 \pi^{2}\right)^{2 / 3}}{10 m_{n} n}\left(n_{n}^{5 / 3}+n_{p}^{5 / 3}\right) \\
& +\left(1-\alpha^{2}\right) v_{s}(n) / n+\alpha^{2} v_{n}(n) / n,
\end{aligned}
$$

where

$$
v_{s}=a_{1} n^{2}+\frac{a_{2} n^{3}}{1+a_{3} n}
$$

and

$$
v_{n}=b_{1} n^{2}+\frac{b_{2} n^{3}}{1+b_{3} n}
$$

are the potential energy densities for symmetric nuclear matter and pure neutron matter, $n_{n}$ and $n_{p}$ are the neutron and proton number densities, $n=n_{n}+n_{p}, \alpha=\left(n_{n}-\right.$ $\left.n_{p}\right) / n$ is the neutron excess, and $m_{n}$ is the neutron mass. Expressions (1)-(3) can well reproduce the microscopic calculations of symmetric nuclear matter and pure neutron matter by Friedman and Pandharipande [26] in the variational method. In this method, the isospin dependence of asymmetric matter EOS is shown to be well approximated by Eq. (1) [27]. (Replacement of the proton mass $m_{p}$ by $m_{n}$ in the proton kinetic energy would make only a negligible difference.) For the later purpose of roughly describing the nucleon distribution in a nucleus, we incorporate into the potential energy densities (2) and (3) a low-density behavior $\propto n^{2}$ as expected from a contact two-nucleon interaction.

A set of expressions (1)-(3) is one of the simplest that reduces to the usual form (4) in the limit of $n \rightarrow n_{0}$ and $\alpha \rightarrow 0$,

$$
w=w_{0}+\frac{K_{0}}{18 n_{0}^{2}}\left(n-n_{0}\right)^{2}+\left[S_{0}+\frac{L}{3 n_{0}}\left(n-n_{0}\right)\right] \alpha^{2} .
$$

Here $w_{0}, n_{0}$, and $K_{0}$ are the saturation energy, saturation density, and incompressibility of symmetric nuclear matter. The parameters $L$ and $S_{0}$ are associated with the densitydependent symmetry energy coefficient $S(n): S_{0}$ is the symmetry energy coefficient at $n=n_{0}$ and $L=3 n_{0}(d S / d n)_{n=n_{0}}$ is the symmetry energy density derivative coefficient (hereafter referred to as the density symmetry coefficient). As the neutron excess increases from zero, the saturation point moves in the density versus energy plane (see, e.g., the dotted lines in Fig. 2). This movement is determined mainly by the parameters $L$ and $S_{0}$. Up to second order in $\alpha$, the saturation energy $w_{s}$ and density $n_{s}$ are given by

$$
w_{s}=w_{0}+S_{0} \alpha^{2}
$$

and

$$
n_{s}=n_{0}-\frac{3 n_{0} L}{K_{0}} \alpha^{2} .
$$

The slope, $y$, of the saturation line near $\alpha=0$ is thus expressed as

$$
y=-\frac{K_{0} S_{0}}{3 n_{0} L} .
$$

We determine the parameters $a_{1}, \ldots, b_{3}$ in such a way that the charge number, charge radius, and mass of stable nuclei calculated in a macroscopic nuclear model constructed in Ref. [2] are consistent with the empirical data. In the course of this determination, we fix $b_{3}$, which controls the EOS of matter for large neutron excess and high density, at $1.58632 \mathrm{fm}^{3}$. This value was obtained by one of the authors [7] in such a way as to reproduce the neutron matter energy of Friedman and Pandharipande [26]. Change in this parameter would make no significant difference in the determination of the other parameters and the final phase diagram.

We describe macroscopic nuclear properties in a way dependent on the EOS parameters $a_{1}, \ldots, b_{3}$ by using a Thomas-Fermi model [2]. The essential point of this model is to write down the total energy of a nucleus of mass number $A$ and charge number $Z$ as a function of the density distributions $n_{n}(\mathbf{r})$ and $n_{p}(\mathbf{r})$ in the form

$$
E=E_{b}+E_{g}+E_{C}+N m_{n} c^{2}+Z m_{p} c^{2},
$$

where

$$
E_{b}=\int d^{3} r n(\mathbf{r}) w\left[n_{n}(\mathbf{r}), n_{p}(\mathbf{r})\right]
$$

is the bulk energy,

$$
E_{g}=F_{0} \int d^{3} r|\nabla n(\mathbf{r})|^{2}
$$


is the gradient energy with adjustable constant $F_{0}$,

$$
E_{C}=\frac{e^{2}}{2} \int d^{3} r \int d^{3} r^{\prime} \frac{n_{p}(\mathbf{r}) n_{p}\left(\mathbf{r}^{\prime}\right)}{\left|\mathbf{r}-\mathbf{r}^{\prime}\right|}
$$

is the Coulomb energy, and $N=A-Z$ is the neutron number. This functional allows us to connect the EOS and the nuclear binding energy through the bulk energy part $E_{b}$. For simplicity we use the following parametrization for the nucleon distributions $n_{i}(r)(i=n, p)$ :

$$
n_{i}(r)= \begin{cases}n_{i}^{\text {in }}\left[1-\left(\frac{r}{R_{i}}\right)^{t_{i}}\right]^{3}, & r<R_{i}, \\ 0, & r \geqslant R_{i} .\end{cases}
$$

This parametrization allows for the central density, halfdensity radius, and surface diffuseness for neutrons and protons separately. To construct the nuclear model in such a way as to reproduce empirical masses and radii of stable nuclei, we first extremized the binding energy with respect to the particle distributions for fixed mass number, five EOS parameters, and gradient coefficient. Next, for various sets of the incompressibility and the density symmetry coefficient, we obtained the remaining three EOS parameters and the gradient coefficient by fitting the calculated optimal values of charge number, mass excess, root-mean-square (rms) charge radius to empirical data for stable nuclei on the smoothed $\beta$ stability line [7]. In the range of the parameters $0<L<160 \mathrm{MeV}$ and $180 \mathrm{MeV}<K_{0}<360 \mathrm{MeV}$, as long as $y \lesssim-200 \mathrm{MeV} \mathrm{fm}^{3}$, we obtained a reasonable fitting to such data (see Fig. 1). As a result of this fitting, the parameters $n_{0}, w_{0}, S_{0}$, and $F_{0}$ are constrained as $n_{0}=0.14-0.17 \mathrm{fm}^{-3}, w_{0}=-16 \pm 1 \mathrm{MeV}$, $S_{0}=25-40 \mathrm{MeV}$, and $F_{0}=66 \pm 6 \mathrm{MeV} \mathrm{fm}^{5}$. We remark that a negative $L$ is inconsistent with the fact that the size of $A=$ $17,20,31$ isobars deduced from the experimental values of the interaction cross section tends to increase with neutron/proton excess [29]. This inconsistency can be seen from Eq. (6), which shows that the saturation density $n_{s}$ increases (and hence the isobar size decreases) with neutron/proton excess for a negative $L$.

We remark that in this range the calculations agree well with a more extended data set of nuclear masses for $A \geqslant 2$ [30] and charge radii for $A \geqslant 50$ [31]. The rms deviations of the calculated masses from the measured values are $\sim 3-5 \mathrm{MeV}$, which are comparable with the deviations obtained from a Weizsäcker Bethe formula, whereas the rms deviations of the calculated charge radii from the measured values are about $0.06 \mathrm{fm}$, which are comparable with the deviations obtained from the $A^{1 / 3}$ law.

Let us summarize the macroscopic nuclear model used here. This model can describe global nuclear properties such as masses and rms radii in a manner that is dependent on the EOS of nuclear matter. One of the important predictions of this model was that the matter radii depend appreciably on the density symmetry coefficient $L$, while being almost independent of the incompressibility $K_{0}$. Although the present macroscopic approach has some limitations in describing the nuclear surface, it is still useful for examining the phase diagram of nuclear matter at subnuclear densities [7].
For the purpose of describing matter in neutron star crusts, we proceed to extend the above-described nuclear model to the case of nuclei of various shapes embedded in a gas of dripped neutrons by following a line of arguments of Ref. [7]. Here we also take into account a gas of electrons as a constituent of matter in the crust and impose $\beta$ stability and charge neutrality in the system.

We consider five phases that consist of spherical nuclei, cylindrical nuclei, planar nuclei, cylindrical bubbles, and spherical bubbles, respectively. Each phase is assumed to be composed of a Coulomb lattice of a single species of nucleus or bubble at a given baryon density $n_{b}$. For the convenience of practical calculations, we adopt the WignerSeitz approximation. In this approximation, a cell in the bcc lattice, including a spherical nucleus or bubble, is replaced by a Wigner-Seitz cell defined as a sphere having the same volume $\left(a^{3}\right)$ and center. We refer to $a$ as the lattice constant. A cylindrical nucleus or bubble having an infinitely long axis and a circular section is taken to be contained in a cylindrical Wigner-Seitz cell having the same axis in place of a cell in the two-dimensional triangular lattice. For a planar nucleus, a Wigner-Seitz cell is identical with a cell in the one-dimensional layered lattice. For the sake of convenience, we redefine the cylindrical and slab Wigner-Seitz cells as a cylinder of height $a$ and base area $a^{2}$ and a slab of thickness $a$ and surface area $a^{2}$, respectively (see Figs. 1 and 2 in Ref. [7]).

For each unit cell, we write the total energy as

$$
W=W_{N}+W_{e}+W_{C},
$$

where $W_{N}, W_{e}$, and $W_{C}$ are the nuclear energy, the electron energy, and the Coulomb energy.

As in Eq. (8), the nuclear energy is again expressed in the density functional form:

$$
\begin{aligned}
W_{N}= & \int_{\text {cell }} d^{3} r\left\{n(\mathbf{r}) w\left[n_{n}(\mathbf{r}), n_{p}(\mathbf{r})\right]\right. \\
& \left.+m_{n} c^{2} n_{n}(\mathbf{r})+m_{p} c^{2} n_{p}(\mathbf{r})+F_{0}|\nabla n(\mathbf{r})|^{2}\right\} .
\end{aligned}
$$

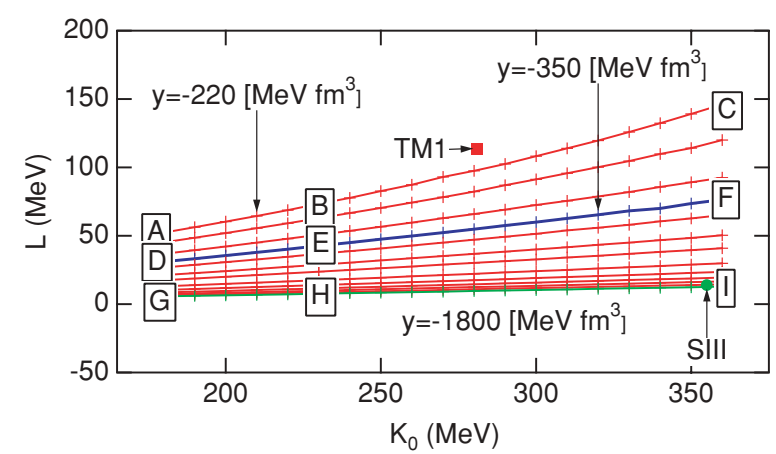

FIG. 1. (Color online) The sets of $\left(L, K_{0}\right)$ (crosses) consistent with the mass and radius data for stable nuclei. The thin lines are lines of constant $y$. The labels A-I denote the sets for which we perform detailed calculations of the ground-state properties of inhomogeneous nuclear matter at subnuclear densities. For comparison, the values calculated from two mean-field models [TM1 (square) and SIII (dot)], which are known to be extreme cases [28], are plotted. The plot shows that our sets of $\left(L, K_{0}\right)$ effectively cover such extreme cases. 


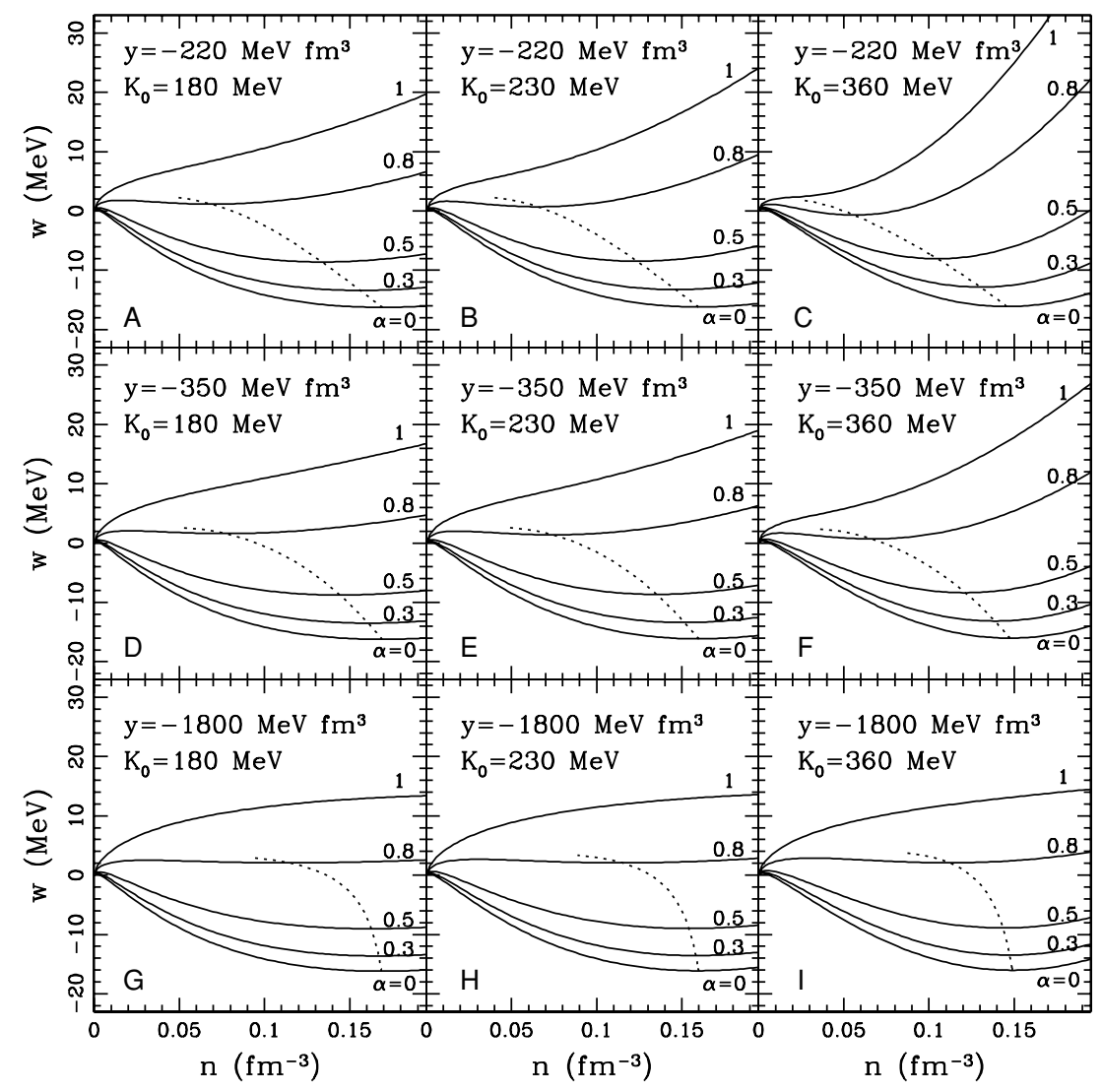

FIG. 2. Energy per nucleon of nuclear matter for the nine sets of $\left(L, K_{0}\right)$ referred to as A-I in Fig. 1. In each panel, the solid lines are the energy at $\alpha=0,0.3,0.5,0.8,1$, and the dotted line is the saturation line.
For a spherical nucleus in vacuum, this expression reduces to $E-E_{C}$ [see Eq. (8)].

The electron energy can be approximated as the energy of an ideal uniform Fermi gas,

$$
\begin{aligned}
\frac{W_{e}}{a^{3}}= & \frac{m_{e}^{4} c^{5}}{8 \pi^{2} \hbar^{3}}\left\{x_{e}\left(2 x_{e}^{2}+1\right)\left(x_{e}^{2}+1\right)^{1 / 2}\right. \\
& \left.-\ln \left[x_{e}+\left(x_{e}^{2}+1\right)^{1 / 2}\right]\right\}
\end{aligned}
$$

with

$$
x_{e}=\frac{\hbar\left(3 \pi^{2} n_{e}\right)^{1 / 3}}{m_{e} c},
$$

where $m_{e}$ is the electron mass and $n_{e}$ is the electron number density that satisfies the charge neutrality condition,

$$
a^{3} n_{e}=\int_{\text {cell }} d^{3} r n_{p}(\mathbf{r}) \text {. }
$$

We remark that $n_{e}$ is so high that we can safely ignore inhomogeneity of the electron density induced by the electron screening of nuclei or bubbles [25] and the Hartree-Fock corrections to the electron energy.

The Coulomb energy is composed of the proton selfCoulomb energy and the lattice energy. We write the Coulomb energy as

$$
W_{C}=\frac{1}{2} \int_{\text {cell }} d^{3} r e\left[n_{p}(\mathbf{r})-n_{e}\right] \phi(\mathbf{r})+\Delta W_{1},
$$

where $\phi(\mathbf{r})$ is the electrostatic potential in a Wigner-Seitz cell, and $\Delta W_{1}$ is the difference of the rigorous calculation [32] for a cell in the bcc (triangular) lattice of spherical (cylindrical) nuclei or bubbles having sharp surfaces from the Wigner-Seitz value, as parametrized in Ref. [7]. We take into account $\Delta W_{1}$, which is a less than $1 \%$ correction, because $\Delta W_{1}$ depends sensitively on the dimensionality of the lattice. (Note that $\Delta W_{1}=0$ for the layered lattice of slab nuclei.)

For nucleon distributions in the Wigner-Seitz cell, we simply generalize the parametrization (12) for a nucleus in vacuum into

$n_{i}(r)= \begin{cases}\left(n_{i}^{\text {in }}-n_{i}^{\text {out }}\right)\left[1-\left(\frac{r}{R_{i}}\right)^{t_{i}}\right]^{3}+n_{i}^{\text {out }}, & r<R_{i}, \\ n_{i}^{\text {out }}, & R_{i} \leqslant r .\end{cases}$

Here $r$ is the distance from the central point, axis, or plane of the unit cell. In the case of nuclei, $n_{p}^{\text {out }}=0$, whereas in the case of bubbles, $n_{p}^{\text {in }}=0$.

We finally determine the equilibrium configuration of the system at given baryon density,

$$
n_{b}=a^{-3} \int_{\text {cell }} d^{3} r n(\mathbf{r}) .
$$

First, for each of the five inhomogeneous phases, we minimize the total energy density $W / a^{3}$ with respect to the eight parameters $a, n_{n}^{\text {in }}, n_{n}^{\text {out }}, n_{p}^{\text {in }}$ (for nuclei) or $n_{p}^{\text {out }}$ (for bubbles), $R_{n}, R_{p}, t_{n}$, and $t_{p}$. This minimization implicitly allows for the stability of the nuclear matter region (the region containing protons) with respect to change in the size, neutron drip, $\beta$ decay, and pressurization. In addition to the five inhomogeneous phases, we consider a uniform phase of $\beta$-equilibrated, neutral nuclear matter. The energy density of this phase is the sum of 
the nucleon part $n w+m_{n} c^{2} n_{n}+m_{p} c^{2} n_{p}$ [see Eq. (1)] and the electron part (15). By comparing the resultant six energy densities, we can determine the equilibrium phase.

\section{EQUILIBRIUM SIZE AND SHAPE OF NUCLEI}

We proceed to show the results for the equilibrium nuclear matter configuration obtained for various sets of the EOS parameters $L$ and $K_{0}$ as shown in Fig. 1. These parameters are still uncertain because they are little constrained from the mass and radius data for stable nuclei [2]. As we shall see, the charge number of spherical nuclei and the density region containing bubbles and nonspherical nuclei have a strong correlation with $L$.

We first focus on spherical nuclei, which constitute an equilibrium state in the low-density region. We calculate the charge number of the equilibrium nuclide as a function of $n_{b}$ for the EOS models A-I as depicted in Fig. 2. Note that the recent GFMC calculations of the energy of neutron matter based on the Argonne v8' potential [15] are close to the behavior of the model E. Hereafter we will thus call the model $\mathrm{E}$ as a typical one. The result is shown in Fig. 3. For densities below $\sim 0.01 \mathrm{fm}^{-3}$, the calculated density dependence of the charge number $Z$ is almost flat, a feature consistent with the results in earlier investigations [1]. More important, the calculated charge number is larger for the EOS models having smaller $L$, and this difference in $Z$ is more remarkable at higher densities.

As we shall see later in this section, this property of $Z$ is related to the tendency that with increasing $L$, the nuclear density decreases while the density of the neutron gas increases. Note that $Z$ is, within a liquid-drop model [1], determined by the size equilibrium condition relating the Coulomb and surface energies in such a way that $Z$ increases with increasing surface tension. Because the Thomas-Fermi model adopted here can be mapped onto a compressible liquiddrop model [2], the present results may well be interpreted in terms of the liquid-drop model. In fact we shall estimate the surface tension from the Thomas-Fermi model as a function of $L$ and discuss how the surface tension depends on the nuclear density and the neutron sea density.

We also note that the density at which the phase with spherical nuclei ceases to be in the ground state is between 0.05 and $0.07 \mathrm{fm}^{-3}$. This result, consistent with the results

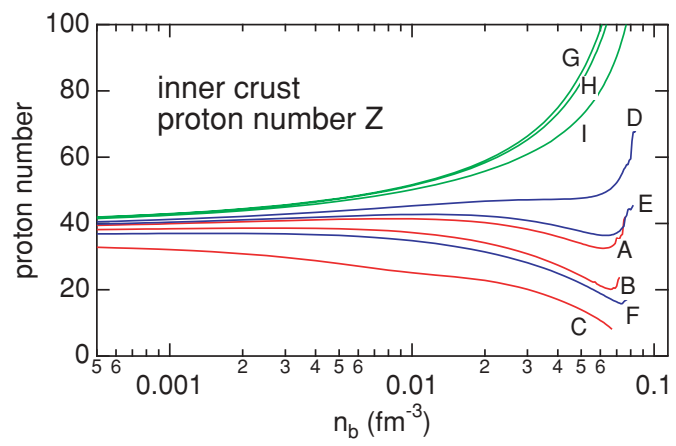

FIG. 3. (Color online) The charge number of spherical nuclei as a function of $n_{b}$, calculated for the EOS models A-I.

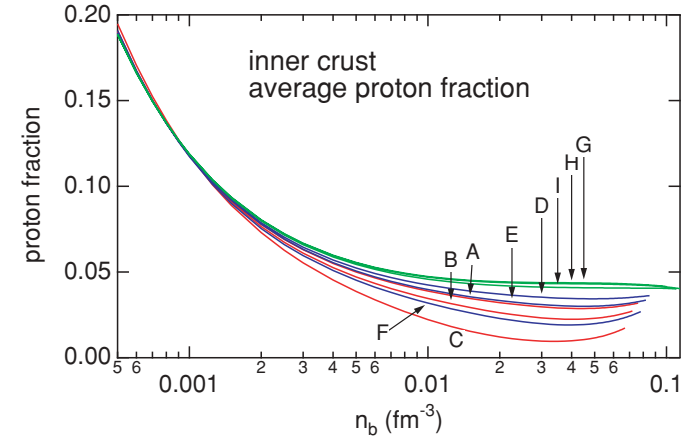

FIG. 4. (Color online) The average proton fraction as a function of $n_{b}$, calculated for the EOS models A-I.

obtained in earlier investigations $[1,7,10]$, will be discussed below in terms of fission instability.

The average proton fraction, which is the charge number divided by the total nucleon number in the cell, is plotted in Fig. 4. We observe that the dependence of the average proton fraction on the EOS models is similar to that of $Z$. We also find that the average proton fraction basically decreases with baryon density. This is a feature coming from the fact that as the baryon density increases, the electron chemical potential increases under charge neutrality and then the nuclei become more neutron-rich under weak equilibrium.

We next consider the density region where bubbles and nonspherical nuclei appear in equilibrium, i.e., the density region of the "pasta" phases. We start with such a density region calculated for the EOS models A-I. The results are plotted in Fig. 5. Except for the model C, we obtain the successive first order transitions with increasing density: sphere $\rightarrow$ cylinder $\rightarrow$ slab $\rightarrow$ cylindrical hole $\rightarrow$ spherical hole $\rightarrow$ uniform matter. A marked correlation of the upper end of the density region with the parameter $L$ can be observed by

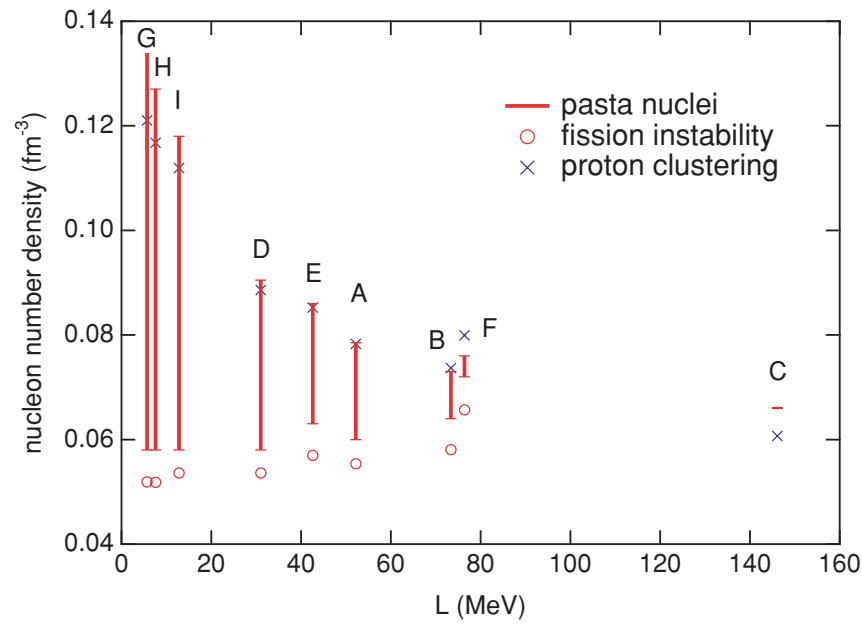

FIG. 5. (Color online) The density region containing bubbles and nonspherical nuclei as a function of $L$, calculated for the EOS models A-I. For comparison, the density corresponding to $u=1 / 8$ in the phase with spherical nuclei and the onset density, $n(Q)$, of proton clustering in uniform nuclear matter, which will be discussed in Sec. IV, are also plotted by circles and crosses, respectively. 

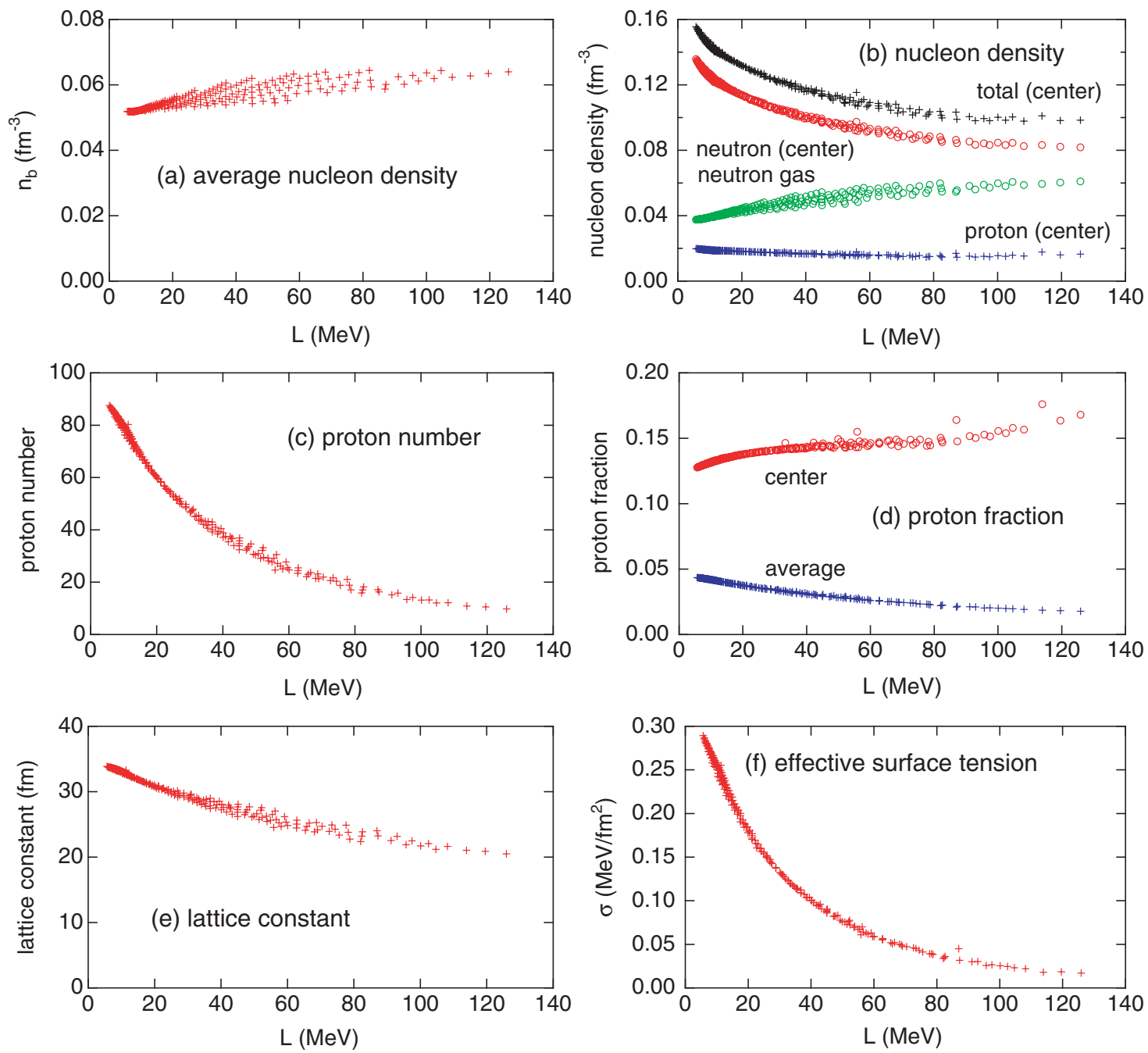

FIG. 6. (Color online) The equilibrium properties of matter with spherical nuclei as a function of $L$, calculated at fixed volume fraction $u=1 / 8$. The baryon density $n_{b}$ (a), the nucleon densities in the center and boundary of the cell (b), the nuclear charge number $Z$ (c), the central and average proton fractions (d), the lattice constant $a(\mathrm{e})$, and the effective surface tension $\sigma$ (f) are plotted.

referring to Fig. 1. This dependence will be examined in detail in the next section.

As can be seen in Fig. 5, the lower end of the density region of the pasta phases has only a weak dependence on the EOS models. To have a closer look at this feature, it is instructive to calculate a density at which spherical nuclei become susceptible to fission-inducing quadrupolar deformations. Within the framework of a liquid-drop model, a spherical liquid-drop in a Wigner-Seitz cell is predicted to undergo such a fission-like instability when the volume fraction $u$, i.e., the ratio of the liquid-drop volume to the cell volume, becomes approximately $1 / 8$ [1]. In such a closely packed situation, the Coulomb self-energy of the liquid-drop amounts to twice the surface energy even under size equilibrium. We note that the density corresponding to $u=1 / 8$ is generally within $\pm 0.01 \mathrm{fm}^{-3}$ of the transition density calculated from the energy comparison between the phases with spherical nuclei and with cylindrical nuclei.

In the present model, we evaluate the volume fraction $u$ as $4 \pi\left(r_{p} / a\right)^{3} / 3$, where $r_{p}$ is the rms radius of the proton distribution multiplied by a factor $\sqrt{5 / 3}$. This is because the proton self-Coulomb energy is relevant to the fission-like instability. At $u=1 / 8$, we calculate the equilibrium properties of matter with spherical nuclei for the parameter sets $\left(L, K_{0}\right)$ included in Fig. 1. The results are plotted as a function of $L$ in Fig. 6. The results for $L>100 \mathrm{MeV}$ are scarce because in this case the pressure of neutron matter is too high for $u$ to amount to $1 / 8$. We remark that the results show only a weak dependence on $K_{0}$.

It is important to note that the baryon density at $u=1 / 8$ is almost flat at $\sim 0.06 \mathrm{fm}^{-3}$ [see Fig. 6(a)]. This is consistent with the lower end of the density region of the pasta phases as depicted in Fig. 5. This magnitude of $n_{b}$ at $u=1 / 8$ can be roughly understood from a simple formula $n_{b} \simeq\left(n_{p}^{\text {in }}+\right.$ $\left.n_{n}^{\text {in }}\right) u+n_{n}^{\text {out }}(1-u)$ with the values of $n_{p}^{\text {in }}, n_{n}^{\text {in }}$, and $n_{n}^{\text {out }}$ in Fig. 6(b). We also note that with increasing $L$, the central density decreases, while the neutron sea density increases. This is natural because both the saturation density of nuclear matter at nonzero neutron excess and the symmetry energy at subnuclear densities decreases with $L$. 
We now turn to the $L$ dependence of the charge number at $u=1 / 8$ [see Fig. 6(c)]. The charge number decreases with $L$. This feature can be understood from the size equilibrium condition within a liquid-drop picture [1]. This condition states that the Coulomb energy of a cell is half as large as the nuclear surface energy. Consequently, the equilibrium charge number squared is proportional to the surface tension and to the nuclear volume. Note that the proton fraction in the nuclear center and the nuclear volume have a relatively weak dependence on $L$ [see Figs. 6(d) and 6(e)]. The surface tension is thus expected to have a similar $L$ dependence to that of the charge number squared, through the dependence on the densities inside and outside the nucleus. From the Thomas-Fermi model, it is reasonable to estimate the effective surface tension as

$$
\sigma=\frac{W_{g}}{2 \pi r_{p}^{2}}
$$

where

$$
W_{g}=\int_{\text {cell }} d^{3} r F_{0}|\nabla n(\mathbf{r})|^{2},
$$

is the gradient energy per cell. This is because in equilibrium, the Coulomb energy of a cell is as large as $W_{g}$ [7], implying that the nuclear surface energy, $\approx 4 \pi \sigma r_{p}^{2}$, is twice as large as $W_{g}$. The surface tension thus estimated basically follows the behavior of $Z^{2}$, as can be seen from Figs. 6(c) and 6(f). The surface tension is generally the function of the neutron excess in the nuclear interior and the densities inside and outside the nucleus [13]. Because the density gradient in the surface region tends to become small for smaller difference between the central density and the neutron sea density, the surface tension decreases with $L$ as shown in Fig. 6(f).

\section{PROTON CLUSTERING IN UNIFORM MATTER}

In this section, we focus on the upper end of the density region of the pasta phases. This upper end corresponds roughly to a density at which uniform nuclear matter neutralized and $\beta$ equilibrated by electrons becomes unstable against proton clustering. In fact, this correspondence can be seen from Fig. 5.

We calculate the onset density of proton clustering by following a line of argument of Baym, Bethe, and Pethick [13]. This density was obtained in Ref. [13] by expanding the energy density functional $E\left[n_{i}(\mathbf{r})\right](i=n, p, e)$ of the system with respect to small density fluctuations $\delta n_{i}(\mathbf{r})$ around the homogeneous state. Whereas the contribution of first order in $\delta n_{i}(\mathbf{r})$ vanishes due to equilibrium of the unperturbed homogeneous system, the second-order contribution can be described in the spirit of the Thomas-Fermi model used here as

$$
E-E_{0}=\frac{1}{2} \int \frac{d^{3} q}{(2 \pi)^{3}} v(q)\left|\delta n_{p}(\mathbf{q})\right|^{2},
$$

where $E_{0}$ is the ground-state energy, $\delta n_{p}(\mathbf{q})$ is the Fourier transform of $\delta n_{p}(\mathbf{r})$, and $v(q)$ is the potential of the effective interaction between protons as given by

$$
v(q)=v_{0}+\beta q^{2}+\frac{4 \pi e^{2}}{q^{2}+k_{\mathrm{TF}}^{2}} .
$$

Here,

$$
\begin{aligned}
v_{0} & =\frac{\partial \mu_{p}}{\partial n_{p}}-\frac{\left(\partial \mu_{p} / \partial n_{n}\right)^{2}}{\partial \mu_{n} / \partial n_{n}}, \\
\beta & =2 F_{0}\left(1+2 \zeta+\zeta^{2}\right), \\
\zeta & =-\frac{\partial \mu_{p} / \partial n_{n}}{\partial \mu_{n} / \partial n_{n}}
\end{aligned}
$$

with $\mu_{n(p)}$ as the neutron (proton) chemical potential, and $k_{\mathrm{TF}} \approx 0.3 n_{e}^{1 / 3}$ is the inverse of the Thomas-Fermi screening length of the electron gas. The effective potential $v(q)$ takes a minimum value $v_{\min }$ at $q=Q$, where

$$
\begin{gathered}
Q^{2}=\left(\frac{4 \pi e^{2}}{\beta}\right)^{1 / 2}-k_{\mathrm{TF}}^{2}, \\
v_{\min }=v_{0}+2\left(4 \pi e^{2} \beta\right)^{1 / 2}-\beta k_{\mathrm{TF}}^{2} .
\end{gathered}
$$

In the energy expansion up to second order in $\delta n_{i}$, the condition that uniform nuclear matter becomes unstable with respect to proton clustering reads $v_{\min }=0$. Generally, $v_{\min }$ is dominated by the bulk contribution $v_{0}$, which decreases with decreasing density (see Fig. 7). This density dependence ensures the presence of a critical density, $n(Q)$, above (below) which the matter is stable (unstable) with respect to proton clustering. Hereafter we will estimate $n(Q)$ without including the gradient and Coulomb contributions to $v_{\min }$, which act to reduce $n(Q)$ only by an amount of order $0.02 \mathrm{fm}^{-3}$ (see Fig. 7 and also Ref. [33]).

The density dependence of $v_{0}$ can be seen by substituting Eq. (1) into Eq. (25). In the limit of $\alpha \rightarrow 1$, to which nuclear matter $\beta$ equilibrated and neutralized by the electron gas is close at subnuclear densities (see Fig. 7), $v_{0}$ behaves roughly as

$$
v_{0} \sim 8 \frac{(\partial \mu / \partial n)_{\alpha=0}}{(\partial \mu / \partial n)_{\alpha=1}} \frac{S(n)}{n},
$$

with the symmetry energy coefficient $S(n)=w_{\alpha=1}(n)-$ $w_{\alpha=0}(n)$. At subnuclear densities, $S(n) / n$ depends only weakly on $n$, whereas the compressibility ratio between pure neutron matter and symmetric nuclear matter, $(\partial \mu / \partial n)_{\alpha=0} /$ $(\partial \mu / \partial n)_{\alpha=1}$, increases almost linearly with density because of the saturation property of the symmetric nuclear matter.

In Fig. 8 we show the results for $n(Q)$ estimated for the parameter sets $\left(L, K_{0}\right)$ included in Fig. 1 . We find that $n(Q)$ decreases with $L$, whereas it does not have a marked dependence on $K_{0}$. The $L$ dependence is correlated with the $L$ dependence of the symmetry energy coefficient $S(n)$ since $S(n)$ acts as a driving force of proton clustering. Note the general tendency that at subnuclear densities, the larger $L$, the smaller symmetry energy coefficient $S(n)$ (see Fig. 7). The proton clustering thus takes place at lower density for larger $L$. Figure 7 also shows that for $n \gtrsim 0.1 \mathrm{fm}^{-3}, S(n)$ becomes larger for larger $L$. This is a feature coming from the empirical relation, $S_{0} \approx 0.075 L+28 \mathrm{MeV}$, derived in Ref. [2]. 

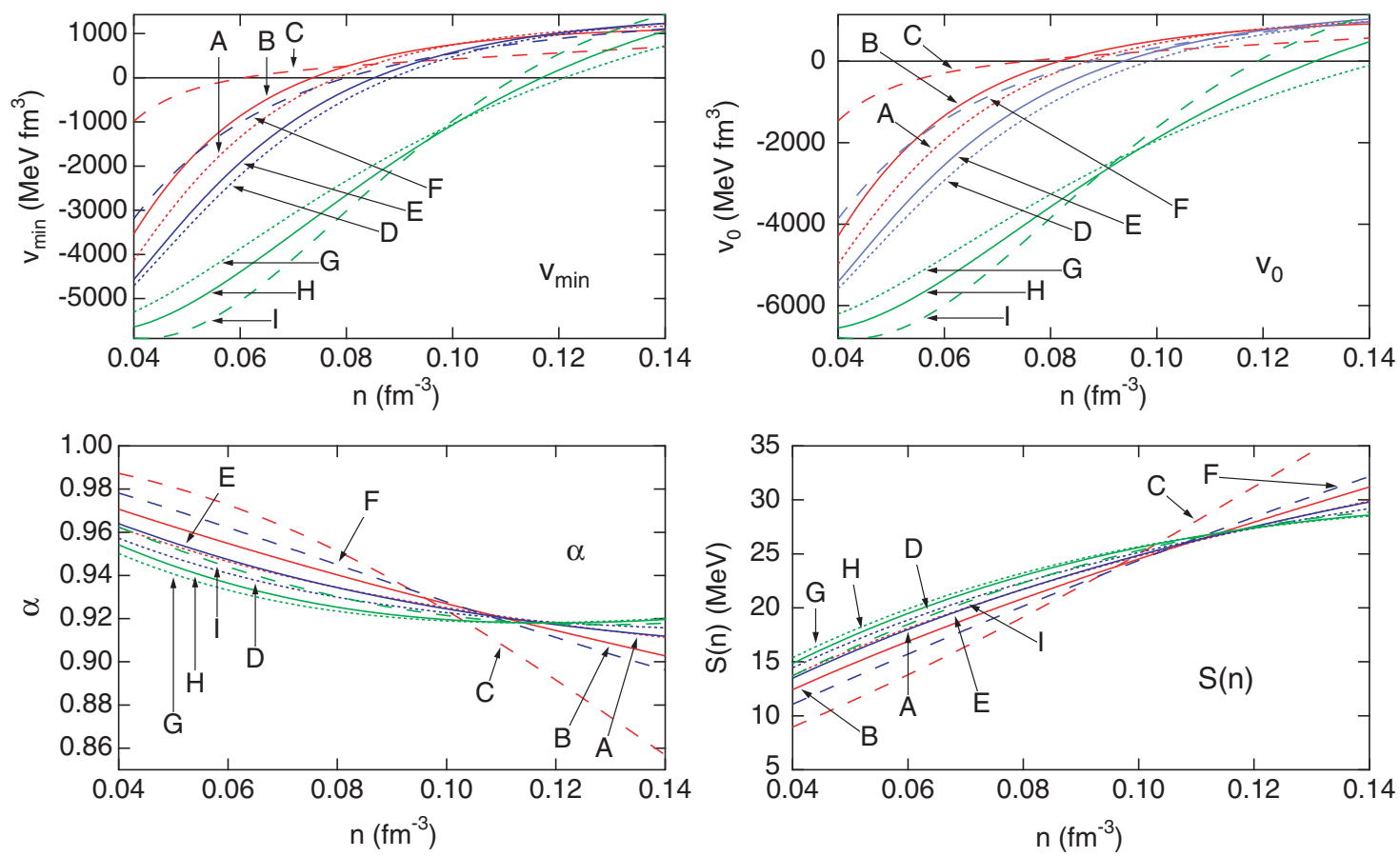

FIG. 7. (Color online) The proton effective potential $v_{\min }$, its bulk part $v_{0}$, neutron excess $\alpha$, and symmetry energy coefficient $S(n)$ as a function of nucleon density, calculated for the EOS models A-I.

We can also observe from Fig. 8 that the difference between $n(Q)$ and the density corresponding to $u=1 / 8$ in the phase with spherical nuclei decreases with $L$ and eventually vanishes near $L=100 \mathrm{MeV}$. This suggests that the density regime of the pasta phases is limited for a large $L$, although for the standard EOS model E, corresponding to $L \simeq 40 \mathrm{MeV}$, it does appear between $\sim 0.06$ and $\sim 0.09 \mathrm{fm}^{-3}$. In our EOS model, a larger value of $L$ implies a harder EOS of pure neutron matter as we shall see below. We thus conclude that the absence of the pasta phases seen in Refs. [6,11,12] from the EOS model with relatively high pressure of neutron matter at subnuclear densities is consistent with our result.

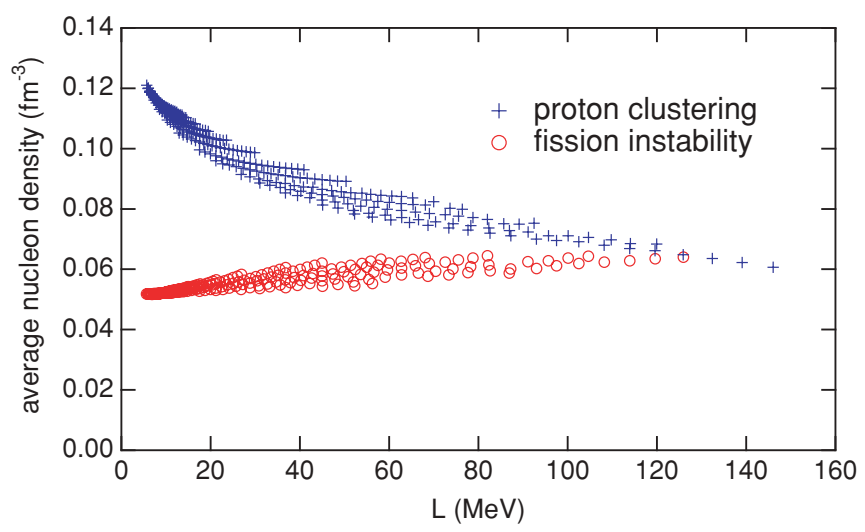

FIG. 8. (Color online) The onset density of proton clustering in uniform nuclear matter as a function of $L$. For comparison, we plot the density corresponding to $u=1 / 8$ in the phase with spherical nuclei, which is taken from Fig. 6(a).
To clarify this consistency, we calculate the pressure of pure neutron matter,

$$
P_{n}=\left.n_{n}^{2} \frac{\partial w}{\partial n_{n}}\right|_{\alpha=1},
$$

for the parameter sets $\left(L, K_{0}\right)$ shown in Fig. 1. The results for $P_{n}$ at $n_{n}=0.1 \mathrm{fm}^{-3}$ are plotted as a function of $L$ in Fig. 9. We find out a roughly linear $L$ dependence of $P_{n}$ at $n_{n}=0.1 \mathrm{fm}^{-3}$. This dependence can be roughly understood by substituting the expansion (4) into Eq. (31) and thereby obtaining

$$
P_{n}=\frac{K_{0}}{9}\left(\frac{n_{n}}{n_{0}}\right)^{2}\left(n_{n}-n_{0}\right)+\frac{L}{3} n_{0}\left(\frac{n_{n}}{n_{0}}\right)^{2} .
$$

We remark that this pressure controls the neutron skin thickness of ${ }^{208} \mathrm{~Pb}$ evaluated within the framework of the Skyrme Hartree-Fock model [34].

We conclude this section by mentioning a relation between the systematic liquid-drop analysis [10] and the present analysis. In Ref. [10], the value of $L$ was fixed at $L=60 \mathrm{MeV}$, and the proton chemical potential in pure neutron matter,

$$
\mu_{p}^{(0)}=\left.\frac{\partial(n w)}{\partial n_{p}}\right|_{\alpha=1},
$$

was changed by a factor of 2 . In the present analysis, however, the value of $L$ was taken between 0 and $160 \mathrm{MeV}$, whereas $\mu_{p}^{(0)}$ depends only weakly on the value of $L$, as shown in Fig. 9, in which the results for $\mu_{p}^{(0)}$ calculated at $n_{n}=0.1 \mathrm{fm}^{-3}$ for the parameter sets $\left(L, K_{0}\right)$ shown in Fig. 1 are plotted as a function of $L$. According to Ref. [10], $\mu_{p}^{(0)}$ plays a role in shifting the density region of the pasta phases without changing its width significantly. We may thus conclude that it is the parameter $L$ that controls the presence of the pasta phases. 

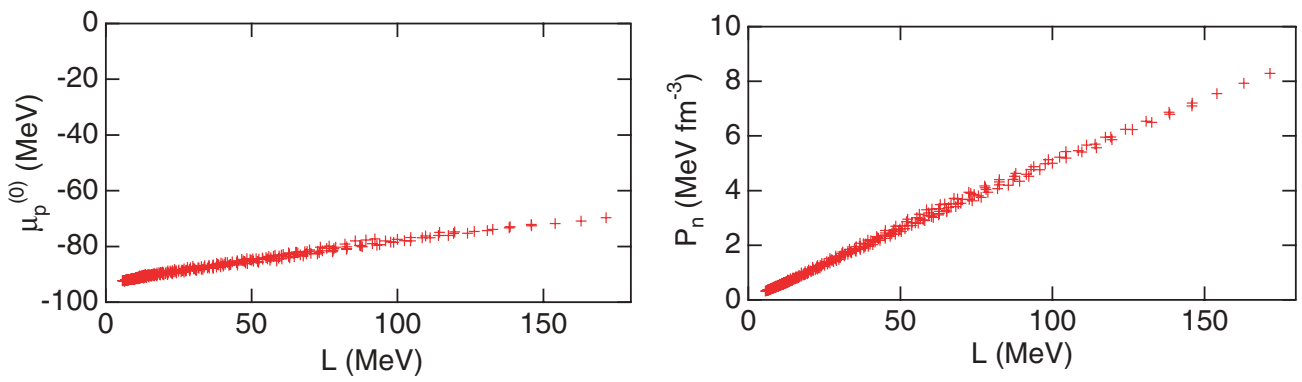

FIG. 9. (Color online) The proton chemical potential and pressure in pure neutron matter of density $0.1 \mathrm{fm}^{-3}$ as a function of $L$.

\section{CONCLUSIONS}

We have analyzed the equilibrium properties of inhomogeneous nuclear matter at subnuclear densities in a way dependent on the density symmetry coefficient $L$ by using a macroscopic nuclear model. We have estimated the upper and lower ends of the density region of the pasta phases from the onset densities of proton clustering in uniform nuclear matter and fission-like instability of spherical nuclei, respectively. We find that the upper end decreases with $L$, whereas the lower end is almost flat at $0.05-0.07 \mathrm{fm}^{-3}$. The former arises from the $L$ dependence of the symmetry energy, whereas the latter can be understood from the volume fraction $u \simeq 1 / 8$ at which spherical nuclei become susceptible to fission-inducing deformations. For a typical EOS model consistent with the GFMC calculations of pure neutron matter, the calculated pasta regime is appreciable. In fact, the pasta regime is predicted to appear when $L \lesssim 100 \mathrm{MeV}$.

The present analysis is the first to attempt a systematic analysis of the pasta region in terms of $L$. However, much care needs to be taken of the interpretation of the results. Although

[1] C. J. Pethick and D. G. Ravenhall, Annu. Rev. Nucl. Part. Sci. 45, 429 (1995).

[2] K. Oyamatsu and K. Iida, Prog. Theor. Phys. 109, 631 (2003).

[3] K. Iida and K. Oyamatsu, Phys. Rev. C 69, 037301 (2004).

[4] D. G. Ravenhall, C. J. Pethick, and J. R. Wilson, Phys. Rev. Lett. 50, 2066 (1983).

[5] M. Hashimoto, H. Seki, and M. Yamada, Prog. Theor. Phys. 71, 320 (1984).

[6] C. P. Lorenz, D. G. Ravenhall, and C. J. Pethick, Phys. Rev. Lett. 70, 379 (1993).

[7] K. Oyamatsu, Nucl. Phys. A561, 431 (1993).

[8] C. J. Pethick, D. G. Ravenhall, and C. P. Lorenz, Nucl. Phys. A584, 675 (1995).

[9] G. Watanabe and H. Sonoda, cond-mat/0502515.

[10] G. Watanabe, K. Iida, and K. Sato, Nucl. Phys. A676, 455 (2000) [Erratum-ibid. A726, 357 (2003)].

[11] K. S. Cheng, C. C. Yao, and Z. G. Dai, Phys. Rev. C 55, 2092 (1997).

[12] F. Douchin and P. Haensel, Phys. Lett. B485, 107 (2000).

[13] G. Baym, H. A. Bethe, and C. J. Pethick, Nucl. Phys. A175, 225 (1971).

[14] P. J. Siemens and V. R. Pandharipande, Nucl. Phys. A173, 561 (1971).
$L$ is the parameter characterizing the expansion of $w$ with respect to $n$ and $\alpha$ around $n=n_{0}$ and $\alpha=0$, the system of interest here is at large neutron excess and at subnuclear densities. The relation between the parameter $L$ and neutron star matter depends on how to parametrize $w$ with respect to $n$ and $\alpha$. It is thus useful to keep in mind that we confined ourselves to expressions (1)-(3) although they are known to be capable of reproducing various existing microscopic calculations of the EOS of uniform nuclear matter.

We have also calculated the charge number $Z$ of spherical nuclei as a function of density for various values of $L$. Generally, the charge number $Z$ becomes smaller for larger $L$, a feature that could be of relevance to the evolution of neutron stars [16]. To make better estimate of $Z$, however, shell and pairing effects should be taken into account.

\section{ACKNOWLEDGEMENT}

This work was supported in part by RIKEN through grant A11-52040.

[15] J. Carlson, J. Morales, V. R. Pandharipande, and D. G. Ravenhall, Phys. Rev. C 68, 025802 (2003).

[16] D. G. Yakovlev and C. J. Pethick, Annu. Rev. Astron. Astrophys. 42, 169 (2004).

[17] R. I. Epstein and G. Baym, Astrophys. J. 387, 276 (1992).

[18] P. B. Jones, Phys. Rev. Lett. 83, 3589 (1999); Mon. Not. R. Astron. Soc. 351, 956 (2004) .

[19] K. Sato, Prog. Theor. Phys. 62, 957 (1979).

[20] P. Haensel and J. L. Zdunik, Astron. Astrophys. 227, 431 (1990).

[21] K. Iida and K. Sato, Astrophys. J. 477, 294 (1997).

[22] F. Montani, C. May, and H. Müther, Phys. Rev. C 69, 065801 (2004).

[23] K. Oyamatsu and M. Yamada, Nucl. Phys. A578, 181 (1994).

[24] P. Magierski and P.-H. Heenen, Phys. Rev. C 65, 045804 (2002).

[25] G. Watanabe and K. Iida, Phys. Rev. C 68, 045801 (2003).

[26] B. Friedman and V. R. Pandharipande, Nucl. Phys. A361, 502 (1981).

[27] I. E. Lagaris and V. R. Pandharipande, Nucl. Phys. A369, 470 (1981).

[28] K. Oyamatsu, I. Tanihata, Y. Sugahara, K. Sumiyoshi, and H. Toki, Nucl. Phys. A634, 3 (1998). 
[29] A. Ozawa et al., Phys. Lett. B334, 18 (1994); L. Chulkov et al., Nucl. Phys. A603, 219 (1996); A. Ozawa et al., ibid. A709, 60 (2002).

[30] G. Audi and A. H. Wapstra, Nucl. Phys. A595, 409 (1995).

[31] H. de Vries, C. W. de Jager, and C. de Vries, At. Data Nucl. Data Tables 36, 495 (1987).
[32] K. Oyamatsu, M. Hashimoto, and M. Yamada, Prog. Theor. Phys. 72, 373 (1984).

[33] K. Iida, G. Watanabe, and K. Sato, Prog. Theor. Phys. 106, 551 (2001) [Erratum-ibid. 110, 847 (2003)].

[34] B. A. Brown, Phys. Rev. Lett. 85, 5296 (2000). 